\title{
Study on the Influence of Hub Motor on Ride Comfort of Electric Vehicle
}

\author{
Weihua Yang ${ }^{a}$, Zifan Fang ${ }^{b}$, Kongde $\mathrm{He}^{\mathrm{c}}$ \\ Mechanic and Power College, China Three Gorges University, Yichang 443002, China \\ aywhuactgu@126.com, bfzf@ctgu.edu.cn, chkd@ctgu.edu.cn
}

Keywords: electric vehicle, hub motor, ride comfort, simulation.

\begin{abstract}
Taking the electric vehicle driven by hub motor as study object, the feature of ride comfort of this type EV is analyzed. The dynamics model of $1 / 4$ vehicle vibration system is established using dynamical theory and state space method,then the simulation of ride comfort of this type EV both under the road excitation and electromagnetic excitation of hub motor is conducted. The simulation results of ride comfort indexes in time-domain are obtained. The simulation results show that the vertical dynamical performance of electric vehicle is changed under the double excitation and the electromagnetic force of hub motor leads to the deterioration of ride comfort. The conclusions have the reference significance to the improvement of the suspension and the determination of vibration control strategy.
\end{abstract}

\section{Introduction}

At present, the energy shortage, environmental pollution and traffic safety problem are becoming more and more serious owing to the rapid growth in vehicle ownership. The development of electric vehicle is an effective way to solve the above problems. Most of all, the electric vehicle independently driven by hub motor has become the future development direction of electric vehicles because of its advantages in unique structure and good dynamic performance [1].

The most typical feature of electric vehicle driven by hub motor is that driving motor is connected with the wheel as a whole, so it is significantly different from the ordinary vehicles both in the structure layout and dynamic performance. In addition to the decline in ride comfort caused by unsprung mass increase because of introduction of hub motor, and what's more, the electromagnetic excitation of hub motor interacts closely with tyre and suspension, meanwhile, electromagnetic characteristics couple to vibration characteristics highly, and consequently cause the adverse effect on the vehicle ride comfort and safety, these problems are worthy of attention and study. Some research on the ride comfort of EV driven by hub motor have been conducted both at home and abroad, but most are establishing the multi-body model based Adams/car software or using Matlab/Simulink software to establish the vibration model, and only consider the road roughness excitation, without considering the influence of vibration of the motor, so these models have few difference with the model of traditional car [2-4]. Furthermore, the relevant documents about motor vibration solve the vibration problem by means of motor design and control study, without taking into account the actual applying and operating conditions of motor, and the research on hub motor which is in the wheel is less.

\section{Characteristic of Ride Comfort of EV Driven by Hub Motor}

Ride comfort is an important performance index which has an important effect on driving performance and the useful life of the vehicle, and also it is the main performance to the top grade car [5]. In the past, studies on ride comfort revolved around the system framework combined with three essential factors which are road, vehicle and people.

Compared with fuel vehicles and EV centralized driven by motor, EV independently driven by hub motor has great difference from the structure form to the driving form, its typical structure is shown in Figure1.Especially due to the introduction of hub motor, the motivation and transmission path of vibration system are changed completely. For this type EV, driving motor is integrated with the wheel, 
and the vibration of the motor is transmitted directly to the body via the wheel, caused the decline of the vehicle ride comfort and safety, therefore, the road roughness and the electromagnetic vibration of hub motor are two main reasons of vehicle body vibration, so the study on ride comfort must consider this change effect on the vibration of system.

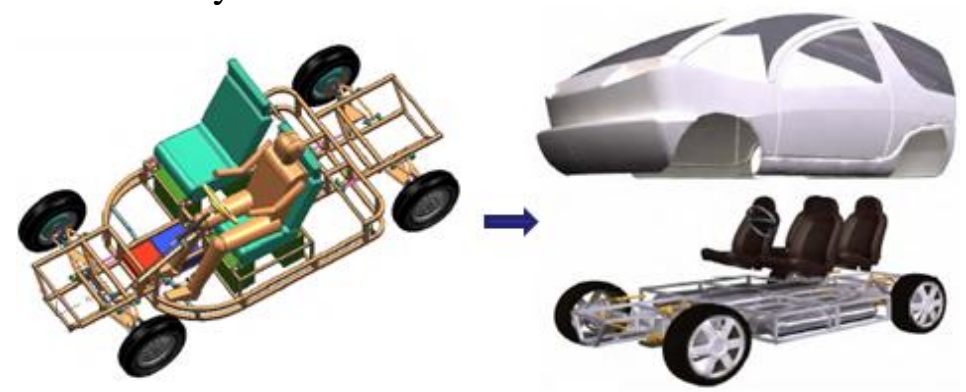

Fig. 1 The typical structure of electric vehicle driven by hub motor

Based on the above analysis, in order to solve the problem of decline of the vehicle ride comfort and safety, vertical dynamic characteristics should be analyzed under the double excitations which are road surface roughness excitation and the electromagnetic vibration of hub motor. Thus, we will focus on a new system diagram about ride comfort analysis of electric vehicle which is shown in figure 2 .

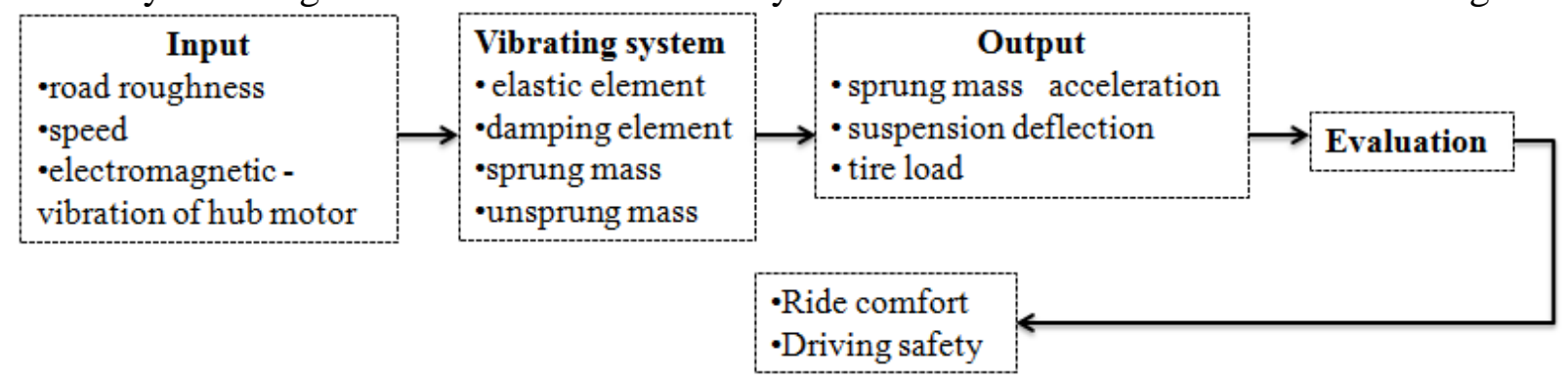

Fig. 2 The new system diagram about ride comfort analysis of electric vehicle

\section{The Dynamics Model of Vibration System of EV under the Double Excitation}

The vehicle dynamics model is the basis of the dynamic analysis. The electric vehicle driven by hub motor is a complex mechanical and electrical vibration system. This type EV can be regarded as the spatial structure of symmetry. According to the focus of study and the special structure of this type electric vehicle, we made some simplifications to the vehicle system before establishing the vibration model $[6,7]$, then electric vehicle vibration system can be simplified as the two degrees of freedom vibration model which is shown in figure 3. It includes both the vertical movement degree of freedom of the sprung mass and the vertical movement degree of freedom of the un-sprung mass. In addition to considering the road roughness, the electromagnetic vibration of hub motor is considered, which is different from the previous model of two degrees of freedom.

In figure $3, m_{d}$ is the mass of hub motor, $m_{l}$ is the un-sprung mass without hub motor, $m_{d}+m_{l}$ is the total un-sprung mass, $m_{s}$ is the total sprung mass, $K$ is the suspension stiffness, $C$ is the suspension damp, $K t$ is the tyre vertical stiffness; $Z_{s}$ and $Z_{u}$ Indicate the vertical displacement of the electric wheel and the body respectively, $Z_{r}$ is road excitation, $F_{d}$ is the electromagnetic vibration of hub motor . 


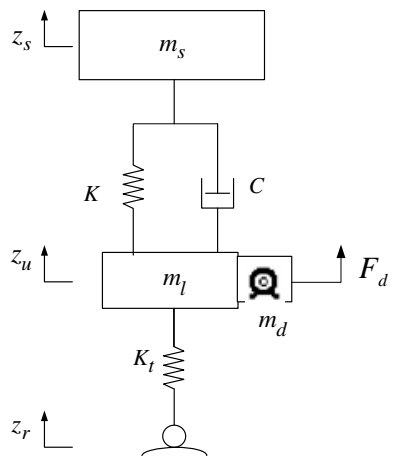

Fig. 3 The dynamic model of one fourth of EV vibration system

According to the Newton's laws of motion, the differential equation of two degrees of freedom vibration system is established as follows:

$$
\begin{aligned}
& m_{s} \ddot{z}_{s}+K\left(z_{s}-z_{u}\right)+C\left(\dot{z}_{s}-\dot{z}_{u}\right)=0 \\
& \left(m_{l}+m_{d}\right) \ddot{z}_{u}+K_{t}\left(z_{u}-z_{r}\right)+K\left(z_{u}-z_{s}\right)+C\left(\dot{z}_{u}-\dot{z}_{s}\right)=F_{d}
\end{aligned}
$$

The State-Space method is applied to compute the above differential equations, so the state -space model can be obtained. The state equation and output equation are as follows:

$$
\dot{X}=A_{2} X+B_{2} u \quad Y=C_{2} X+D_{2} u
$$

Where, $X=\left[x_{1}, x_{2}, x_{3}, x_{4}\right]^{T}=\left[z_{s}, \dot{z}_{s}, z_{u}, \dot{z}_{u}\right]^{T}$ is state variable, $x_{1}=z_{s}$ is the vertical displacement of the sprung mass, $x_{2}=\dot{z}_{s}$ is the vertical velocity of the sprung mass, $x_{3}=z_{u}$ is the vertical displacement of un-sprung mass, $x_{4}=\dot{z}_{u}$ is the vertical velocity of un-sprung mass. $u=\left[z_{r}, F_{d}\right]^{T}$ is the input vector , and $Y=\left[y_{1}, y_{2}, y_{3}\right]^{T}=\left[\ddot{z}_{s}, z_{s}-z_{u}, z_{u}-z_{r}\right]^{T}$ is the output vector, $y_{1}=\ddot{z}_{s}$ is the vertical acceleration of sprung mass, $y_{2}=z_{s}-z_{u}$ is the suspension deflection, $y_{3}=z_{u}-z_{r}$ is the deformation of tyre. $A_{2}$ is the system matrix, $B_{2}$ is the output matrix, $C_{2}$ is the control matrix, $D_{2}$ is the direct transfer matrix.

\section{Ride Comfort Simulation under the Double Excitation of Road and Hub Motor}

Computer programming based on the Matlab software platform is conducted. The program includes the state space model of 1/4 vehicle vibration system, the model of road spectrum and the calculation model of hub motor electromagnetic force .During the simulation, four-order Rung Kutter algorithm is used to solute the state space model of vehicle vibration system, calculating step is 0.005 , and length of the sample is $0.005 \mathrm{~s}$

In order to compare the difference in ride comfort under the double excitation and single road excitation, Ride comfort simulation under the single road excitation is made. Finally, the ride comfort simulation results in time domain are shown in the table 1 and the figure 4 (a) - (c).

Table 1 Statistics about Values of Performance Indexes In Time Domain

\begin{tabular}{|c|c|c|c|c|c|c|}
\hline \multirow{2}{*}{} & \multicolumn{2}{|c|}{ sprung mass acceleration } & \multicolumn{2}{c|}{ suspension deflection } & \multicolumn{2}{c|}{ tyre dynamic displacement } \\
\cline { 2 - 6 } & RMS & maximum & RMS & maximum & RMS & maximum \\
\hline (1)single excitation & 0.8701 & 3.0092 & 0.0053 & 0.0172 & 0.0021 & 0.0074 \\
\hline $\begin{array}{c}\text { (2)double excitation } \\
\text { change rate of (2) to } \\
\text { (1) }\end{array}$ & 0.9605 & 3.3938 & 0.0054 & 0.0188 & 0.0023 & 0.0076 \\
\hline
\end{tabular}




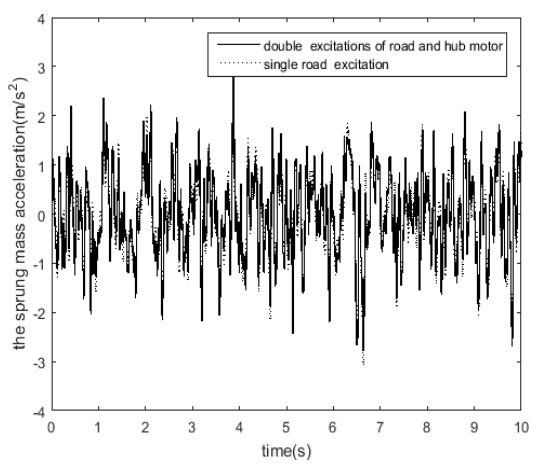

(a) sprung mass acceleration

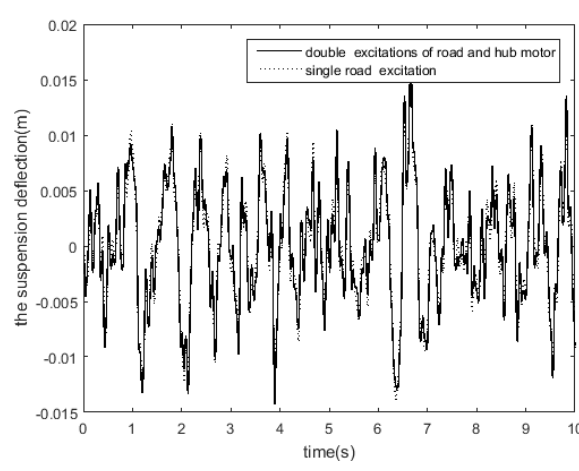

(b) suspension deflection

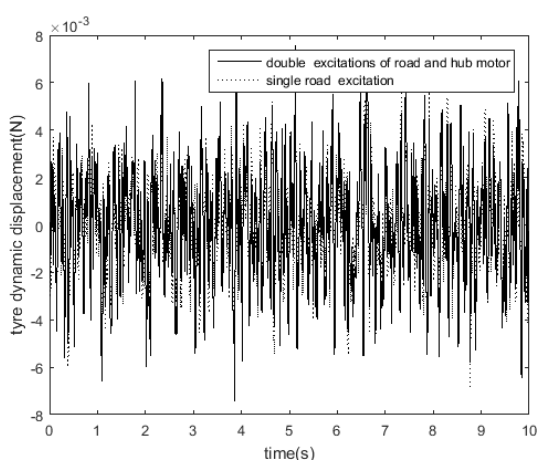

(c) tyre dynamic displacement

Fig. 4 The ride comfort simulation result in time domain

As it can be seen from Figure 4 , the vehicle body acceleration, suspension deflection and tyre dynamic displacement under the double excitation have been increased with varying degrees of growth compared with the values under the single excitation. Furthermore, as it can be seen from Table 1 , the electromagnetic excitation has the most obvious effect on the vehicle body acceleration. From the RMS statistical results of three performance indexes about ride comfort, it can be shown that the influence on three performance indexes is different, and the impact of electromagnetic excitation on vibration responses is as follows: the vehicle body acceleration, tyre dynamic displacement and suspension deflection respectively.

\section{Conclusion}

In view of the above analysis, the conclusions which we can draw are as followed: the vertical dynamic performance has changed obviously due to the electromagnetic excitation of hub motor; The electromagnetic characteristics and vibration characteristics of this type EV have been coupled highly, which is the main reason leading to the decline of ride comfort and driving safety. How to reduce or even eliminate the above adverse effects will be the key problem to be solved in the development of EV driven by hub motor in the future.

\section{References}

[1]. Watts A, Vallance A, Whitehead A, et al. The technology and economics of in-wheel motors.SAE International Journal of Passenger Cars-Electronic and Electrical Systems, Vol. 3 (2010) No. 2, p. 37-54.

[2]. Chen Qiu-ming ,Chen Yong. Study on Theory of Electro-Magnetic Vibration and Noise of a Permanent Magnet Synchronous Motor. Journal of Micro-motor, Vol. 41 (2013) No. 8, p. 1-5

[3]. XIANG Long-yang,Zuo Shu-guang,He Lv-chang,etc. The vibratory behavior analysis of stator assembly of brushless direct current motor. Journal of North China Electric Power University, Vol. 38 (2011) No. 6, p. 23-28

[4]. Wang Li-lan. Researches on Behavior of Vibration and Noise in Axial Flux Permanent Magnet Motor (D). Shenyang: Shenyang University of Technology(2008)

[5]. Yu Zhi-sheng. Automobile theory (the Fifth Edition). Beijing: mechanical industry press(2011)

[6]. Fang Zi-fan. Study on Control Method of Automotive Semi-active Suspension System Based On MR damper(D),..Chongqing: Chongqing University(2006)

[7]. Tan Di. Dynamics and Structure Optimization of the In-wheel Motor System with Rubber Bushing(D). Guangzhou: South China University of Technology(2013) 